\title{
Simulations interactives de convertisseurs en électronique de puissance
}

\author{
Jean-Jacques HUSELSTEIN, Philippe ENRICI \\ Laboratoire d'Électrotechnique de Montpellier (LEM) - Université Montpellier II, \\ CC079, Place Eugène Bataillon, 34095 MONTPELLIER CEDEX 5, \\ huselstein@univ-montp2.fr, enrici@univ-montp2.fr
}

\begin{abstract}
RÉSUMÉ Cet article présente un ensemble d'applications interactives de simulation fonctionnant en « temps réel apparent » destinées à l'enseignement de l'électronique de puissance. Nous avons étudiés les convertisseurs suivant : hacheur série 1 quadrant, hacheur 2 quadrants, hacheur 4 quadrants, onduleur monophasé, hacheur parallèle, alimentation FLYBACK, redressement sur charge capacitive, .... Ces VI (LabVIEW Virtual Instrument) de simulation peuvent servir comme aide au dimensionnement, mais leur objectif reste essentiellement pédagogique. Il s'agit de retrouver sur un écran d'ordinateur (ou sur l'écran de la salle de cours avec un vidéo projecteur) ce que l'on pourrait voir sur un oscilloscope avec un vrai montage. L'intérêt par rapport à un simulateur classique est de pouvoir modifier n'importe quel paramètre, grandeur de commande ou valeur de composant, et de visualiser immédiatement l'effet produit. Lors d'un cours cela permet à l'enseignant de monter les fonctionnements des convertisseurs de l'électronique de puissance de manière plus facile et plus vivante qu'avec une succession de « transparents » plus ou moins figés. Les étudiants peuvent ensuite poursuivre l'étude de ces montages dans les salles d'informatiques ou à leur domicile, ces applications étant mises à leur disposition sur une page internet.
\end{abstract}

Mots clés : Simulation, LabVIEW, onduleur, hacheur, MLI, commande, puissance, modulation.

\section{INTRODUCTION}

Il est habituel d'utiliser un simulateur électrique de type circuit pour étudier les montages de l'électronique de puissance. Pour nos enseignements nous utilisons couramment le logiciel PSIM. La version de démonstration de ce programme suffit déjà pour étudier beaucoup de structures de base de l'électronique de puissance et sa gratuité permet de l'installer sur toutes les machines des salles informatiques et autorise aussi les étudiants à l'utiliser à leur domicile.

Les limitations d'un simulateur circuit dans une phase d'apprentissage résident dans le fait que lorsque l'on veut visualiser les effets des variations d'un paramètre de fonctionnement (par exemple la fréquence de découpage, la valeur de l'inductance de lissage, ...) il est nécessaire d'ouvrir l'éditeur de schéma, de modifier la valeur puis de relancer une nouvelle simulation. Si l'on souhaite monter les effets de nombreux paramètres ce mode d'utilisation devient rapidement lent et fastidieux.

L'idée de faire un simulateur interactif avec une apparence de temps réel nous a été inspirée par les travaux de Kolar et Drofenik [1], [2] mis à disposition sur leur site web «IPES-circuit» [3]. Ce site internet est un module d'enseignement dédié aux circuits de bases de l'électronique de puissance. Les interfaces de simulation sont des applet Java que l'utilisateur utilise et paramètre par des déplacements de souris. Les résultats de simulation sont visibles «instantanément» sous formes de courbes temporelles. Accessoirement il est également possible de visualiser à chaque instant le chemin parcouru par les courants sur le schéma en déplaçant un curseur sur les oscillogrammes.

Ce module de simulation est très complet et vraiment remarquable. Cependant nous avions envie de créer nos propres outils de simulation pour les faire correspondre à nos propres objectifs pédagogiques. Nous souhaitions des simulations interactives visualisées en «temps réel» mais avec des résultats quantitatifs précis et avec un choix de tous les paramètres de fonctionnement effectué par l'utilisateur. Ceci autant pour les paramètres de commande (fréquence de découpage, rapport cyclique, mode de commande, ...) que pour les valeurs des éléments passifs (inductance de lissage, capacité de filtrage, résistance de charge, ...).

Nous avons donc réalisé quelques applications interactives de simulation « temps réel » de circuits simples de l'électronique de puissance. Nous avons utilisé LabVIEW comme langage de programmation. Ceci parce que nous en disposions et étions habitués à l'utiliser et surtout parce qu'il est aisé de réaliser des interfaces graphiques très performantes (pour les affichages temporels et pour les réglages de paramètres). Nous avions précédemment déjà réalisé avec LabVIEW des programmes de simulation dans cet état d'esprit sur le fonctionnement en régimes permanent de transformateurs et de machines synchrones en vitesse variable [4].

La programmation graphique de LabVIEW est relativement aisée et rapide (par rapport à une programmation en langage $\mathrm{C}$ par exemple). La vitesse de calcul est très élevée et donne une excellente « fluidité » aux simulations. 


\section{HACHEURS}

\subsection{Hacheur série 1 quadrant et 2 quadrants}

Ce VI permet de simuler les hacheurs série 1 quadrant (non réversible, un transistor et une diode) ou 2 quadrants (réversible en courant, deux transistors et deux diodes). Le choix du type de hacheur se fait par un « commutateur» sur la face avant.

La durée de l'intervalle de simulation (choisie par l'utilisateur) correspond à la largeur d'un écran d'affichage. L'utilisateur peut également modifier le nombre de points de calcul correspondant à cette durée. La simulation est lancée lors du démarrage du VI (appui sur la flèche dans la barre de contrôle). La simulation correspondant à une largeur d'écran d'affichage dure de quelques ms à quelques dizaines de ms selon la puissance de l'ordinateur utilisé et le nombre de points de calculs demandé. Lorsque tous les points de l'intervalle de simulation ont été calculés le calcul reprend à $\mathrm{t}=0$. Si la réinjection des valeurs finales est demandée (bouton sur la face avant) les valeurs finales des grandeurs d'états (tensions aux bornes du condensateur et courant dans l'inductance) sont prises comme valeurs initiales pour la simulation suivante qui démarre dès que les calculs du premier intervalle (largeur écran) sont terminés. Remarque : en réalité ce ne sont pas les valeurs finales qui sont prises en compte mais celles correspondant au plus grand multiple entier de la période de découpage compris dans l'intervalle de simulation. Ceci principe donne l'impression de fonctionner avec un oscilloscope sur un montage réel avec une fréquence de rafraîchissement de plusieurs dizaines de Hz.

Tous les paramètres de fonctionnement (fréquence de découpage, rapport cyclique, tension d'entrée, valeurs des éléments passifs du circuit $\mathrm{L}, \mathrm{C}$ et $\mathrm{R}$ ) peuvent être ajustés librement par l'utilisateur pendant toute la durée de la simulation.

Si la réinjection des valeurs finales n'est pas demandée (bouton sur la face avant) et lorsque tous les points de l'intervalle de simulation ont été calculés le calcul reprend à $\mathrm{t}=0$. Dans ce cas on étudie le régime transitoire avec des valeurs de tension et courant initiales nulles.

Le diagramme temporel représente les tensions et courants les plus représentatifs du fonctionnement du hacheur : tension de sortie $v_{S}$ avant filtrage, courant $i_{L}$ dans l'inductance de lissage et tension $\mathrm{E}$ aux bornes du condensateur de filtrage de sortie.

Le diagramme de conductions des interrupteurs $\left(T_{1}\right.$, $\mathrm{D}_{1}, \mathrm{~T}_{2}$ et $\mathrm{D}_{2}$ ) est automatique généré durant la simulation. Il permet de mieux comprendre le fonctionnement d'un hacheur réversible en courant ou la conduction en régime discontinu d'un hacheur non réversible.

Les plages des échelles d'affichage (temps, tension et courant) peuvent être librement modifiées par l'utilisateur en entrant directement de nouvelles valeurs pour la première et la dernière étiquette de chaque échelle.

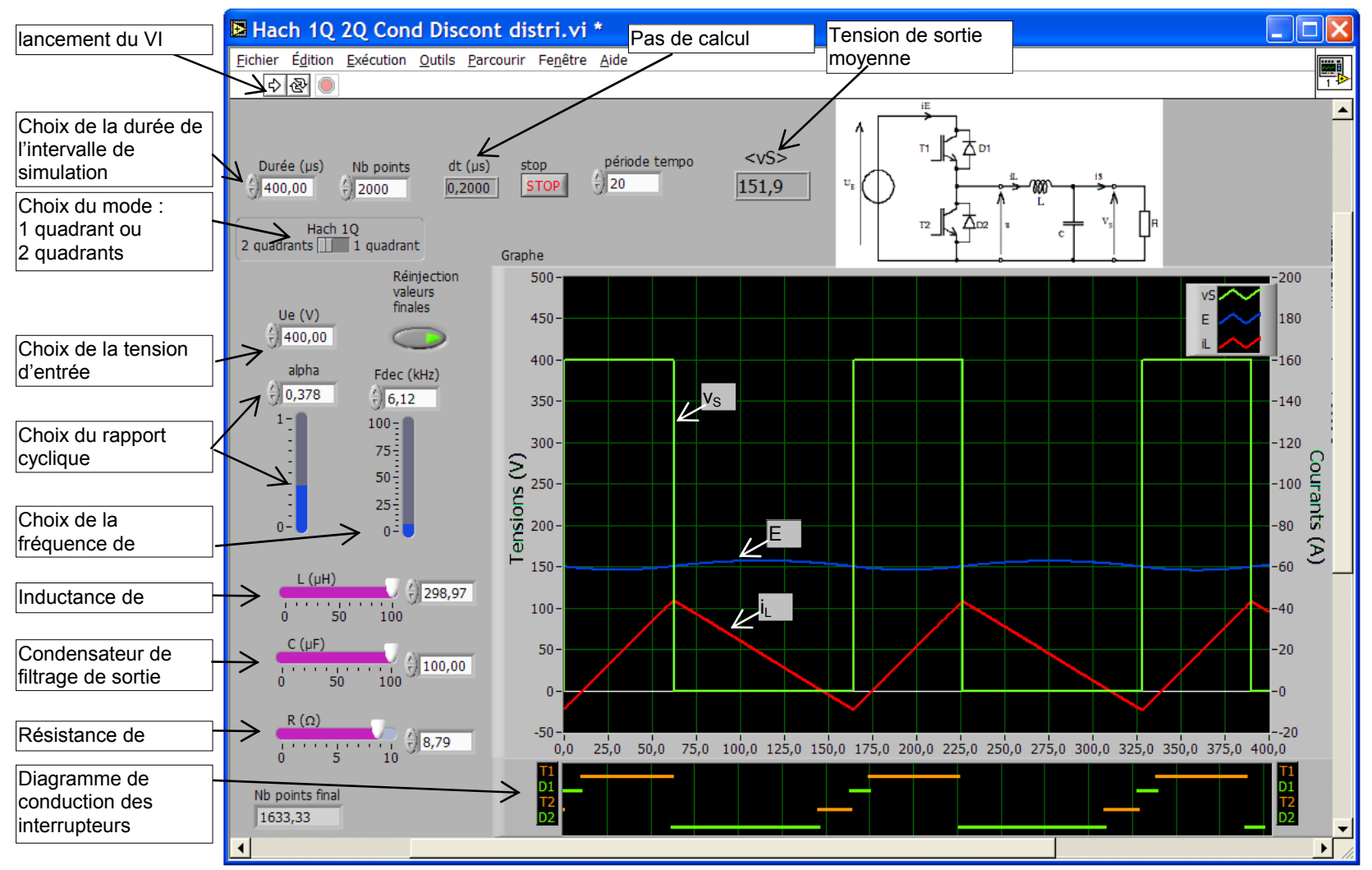

Fig. 1 : Hacheur série (1 quadrant ou 2 quadrants). Commandes de face avant. 


\subsection{Fonctionnement du simulateur}

\subsubsection{Principe}

Le calcul numérique est basé sur une méthode très simple d'intégration discrète des variables d'états du circuit avec une topologie variable. Dans le cas des hacheurs et onduleurs de tension monophasés par exemple, il s'agit du courant de l'inductance de lissage et de la tension aux bornes du condensateur de filtrage de sortie. Les états ouvert ou fermé des semiconducteurs sont déterminés par le circuit de commande et la variable d'état représentant le courant dans l'inductance. Ces états des interrupteurs permettent de connaître la tension aux bornes de l'inductance et le courant traversant le condensateur. Ces valeurs de tension et de courant sont utilisées pour l'intégration discrète déterminant les valeurs des grandeurs d'état pour le pas de calcul suivant.

\subsubsection{Exemple : hacheur parallèle}

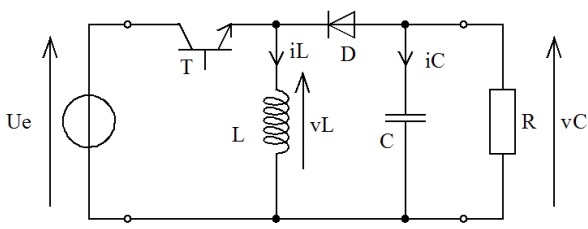

Fig. 2 : Schéma hacheur parallèle.

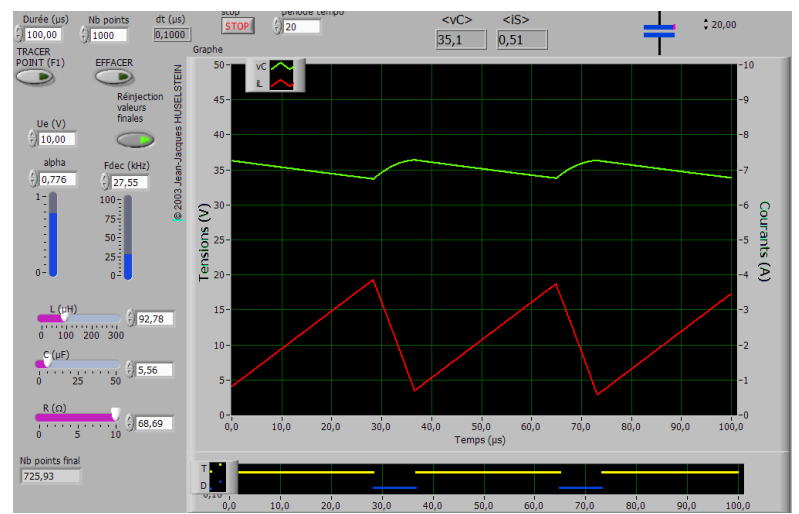

Fig. 3 : Face avant Hacheur parallèle.

Les équations générales sont :

$$
i_{L_{n+1}}=i_{L_{n}}+\frac{v_{L_{n}}}{L} d t \quad v_{C_{n+1}}=v_{C_{n}}+\frac{i_{C_{n}}}{C} d t
$$

Calcul des valeurs de $\mathrm{v}_{\mathrm{L}}$ et de $\mathrm{i}_{\mathrm{C}}$ :

- Quand T est passant (donc la diode D bloquée) :

$$
v_{L}=U_{E} \quad i_{C}=-\frac{v_{C}}{R}
$$

- Quand T est bloqué :

si $i_{L}>0$ (diode passante) $\Rightarrow v_{L}=v_{C}$ et $i_{C}=-i_{L}-\frac{v_{C}}{R}$

si $i_{L}=0$ (diode bloquée) $\Rightarrow v_{L}=0$ et $i_{C}=-\frac{v_{C}}{R}$

\subsubsection{Exemple : alimentation à découpage Flyback}

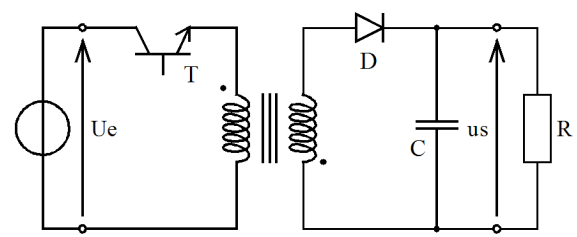

Fig. 4 : Alimentation à découpage Flyback

Équations générales :

On considère l'inductance magnétisante ramenée au secondaire

$$
i_{L_{n+1}}=i_{L_{n}}+\frac{v_{L_{n}}}{L} d t \quad v_{C_{n+1}}=v_{C_{n}}+\frac{i_{C_{n}}}{C} d t
$$

Valeurs de $\mathrm{v}_{\mathrm{L}}$ et de $\mathrm{i}_{\mathrm{C}}$ :

- Quand T est passant :

$$
v_{L}=\frac{n_{2}}{n_{1}} U_{E} \quad i_{C}=-\frac{v_{C}}{R}
$$

- Quand T est bloqué :

$$
\begin{aligned}
& \text { si } i_{L}>0(\text { diode passante }) \Rightarrow v_{L}=v_{C} \text { et } i_{C}=-i_{L}-\frac{v_{C}}{R} \\
& \text { si } \left.i_{L}=0 \text { (diode bloquée }\right) \Rightarrow v_{L}=0 \text { et } i_{C}=-\frac{v_{C}}{R}
\end{aligned}
$$

\subsection{Filtrage sur les structures hacheur}

L'écran ci-dessous (Fig. 5) représente un fonctionnent en régime transitoire d'un hacheur 2 quadrants (obtenu en supprimant la réinjection de valeurs finales).

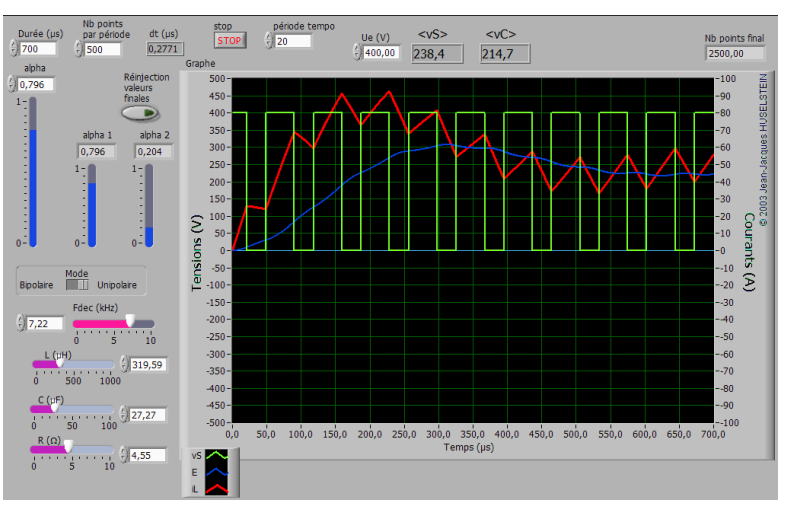

Fig. 5 : Hacheur 2 quadrants en phase transitoire.

\subsection{Hacheurs série entrelacés}

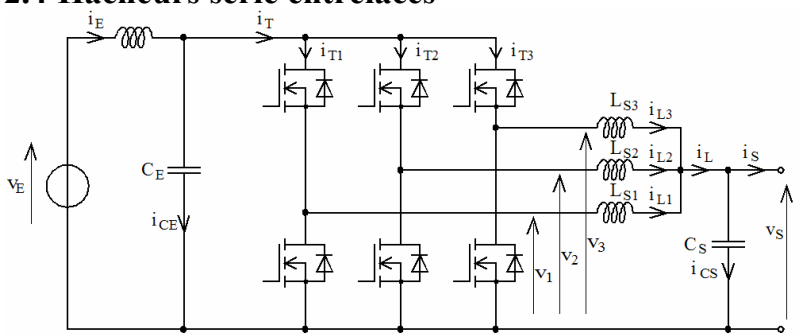

Fig. 6 : Hacheurs série entrelacés. 
La face avant suivante (Fig. 7) représente les chronogrammes de fonctionnement de trois hacheurs série entrelacés (Fig. 6). Les trois hacheurs sont commandés avec la même fréquence de découpage et le même rapport cyclique.

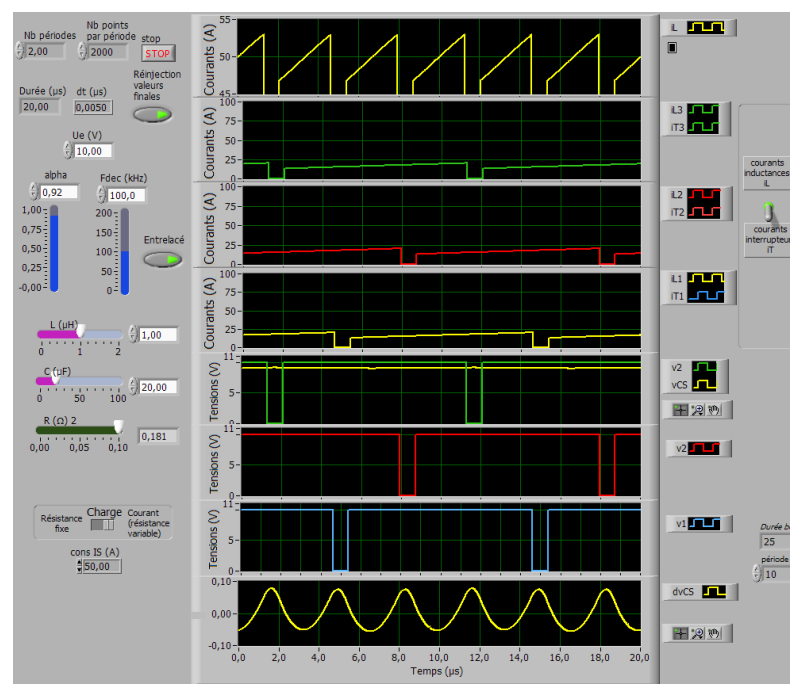

Fig. 7 : Entrelacement de trois hacheurs série.

Ce fonctionnement est aujourd'hui couramment utilisé dans les convertisseurs continu-continu d'alimentation de circuits électroniques sous faible tension et fort courant (par exemple un convertisseur 3,3V vers $1,2 \mathrm{~V}$ $80 \mathrm{~A}$ pour un Pentium IV).

\subsection{Hacheur 4 quadrants : modes de commande}

Nous avons réalisé deux VI pour les hacheurs quatre quadrants. Le premier (non exposé ici) est plutôt orienté pour l'étude du filtrage avec prise en compte du mode de commande bipolaire ou unipolaire. Le deuxième, dont la face avant est présentée ci-dessous (Fig. 8), a été plutôt pensé pour une étude globale du fonctionnement au niveau des valeurs moyennes de tension et de courants, d'entrée et de sortie, ainsi que de la puissance convertie. Sa particularité est de visualiser un schéma équivalent partiel pour chacune des phases de fonctionnement, le schéma partiel affiché correspondant à l'instant t pointé par le curseur. Il est ainsi aisé d'expliquer les différentes phases de conduction des 8 semi-conducteurs de puissances (4 transistors et 4 diodes) qui composent le convertisseur. Lors de la simulation il est bien sûr possible de modifier tous les paramètres de fonctionnement, qu'il s'agisse de la commande, de la valeur de l'inductance de lissage ou du courant moyen absorbé par la charge. D'autre part les grandeurs de fonctionnement globales $\mathrm{du}$ hacheur en valeurs moyennes sont également affiché dans un plan $\mathrm{V}=\mathrm{f}(\mathrm{I})$

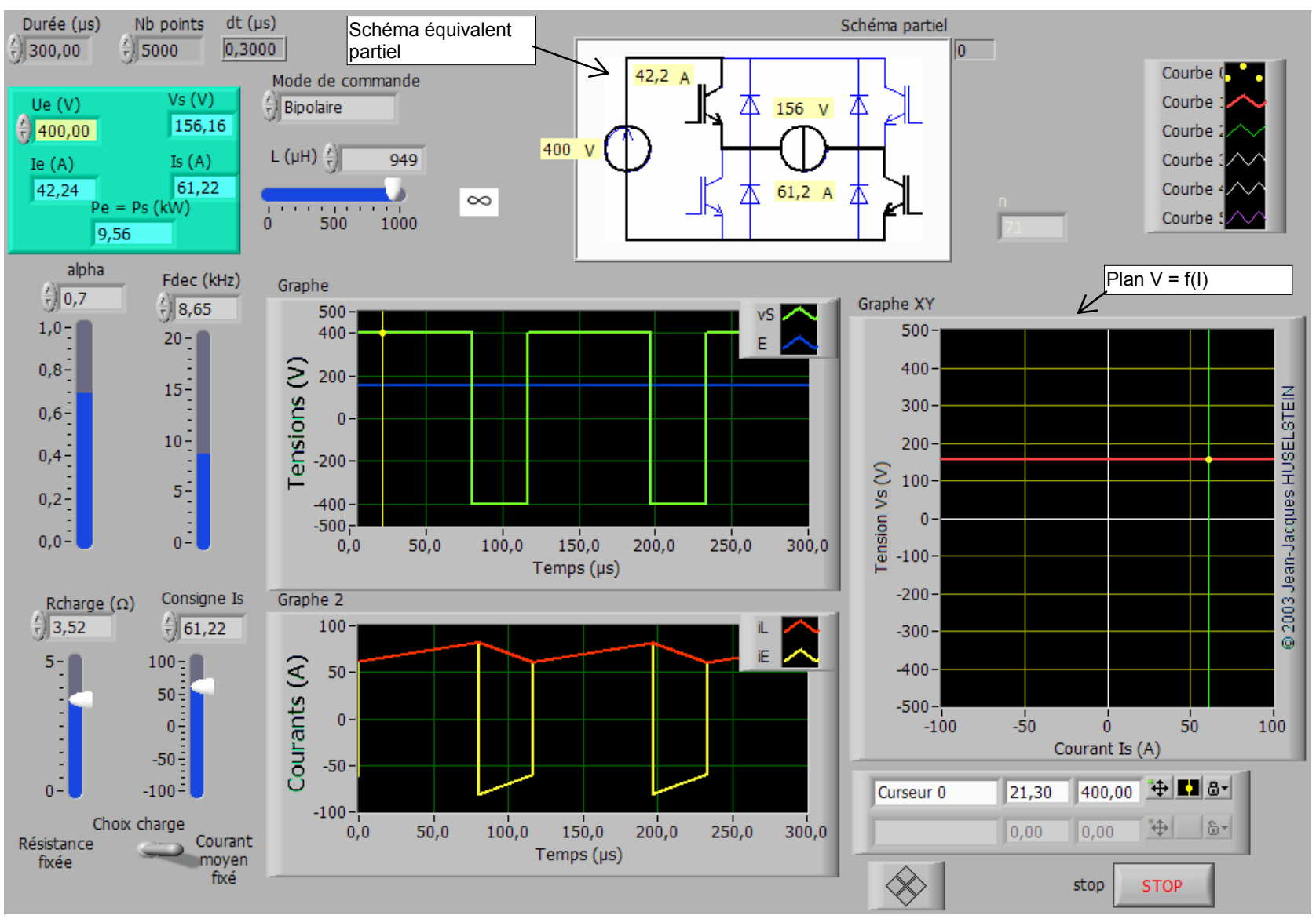

Fig. 8 : Hacheur 4 quadrants. 


\section{ONDULEURS}

\subsection{Onduleurs monophasés}

Les onduleurs de tension monophasés sont des hacheurs 4 quadrants dont le rapport cyclique est modulé sinusoïdalement autour d'une valeur moyenne égale à 0,5 . Nous avons donc simplement modifié en conséquence le hacheur 4 quadrants déjà présenté. Le choix du mode de commande, bipolaire ou unipolaire, est conservé. Il est possible de choisir la fréquence de modulation BF et la profondeur de modulation. En plus des grandeurs de sortie, le courant d'entrée, avant et après filtrage, est également affiché.

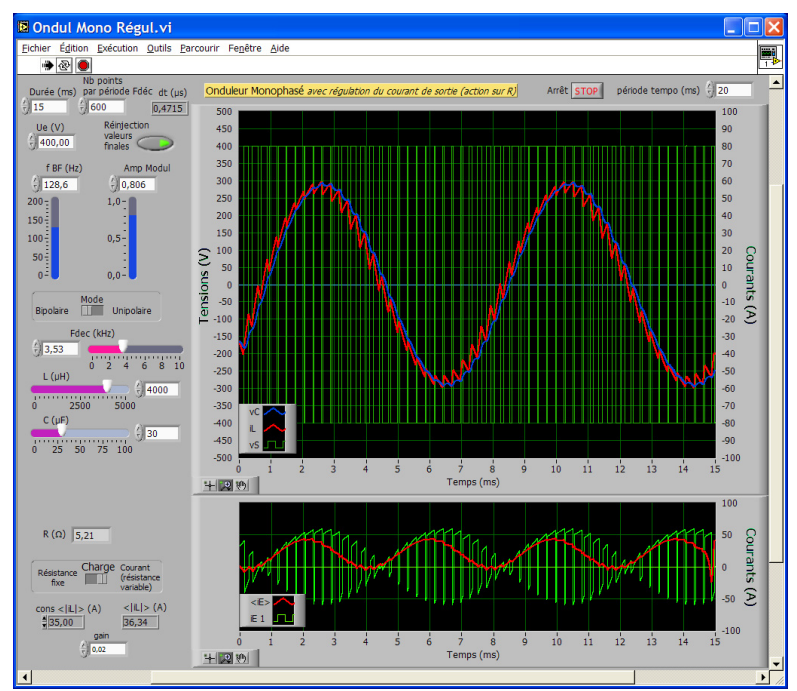

Fig. 9 : Onduleur monophasé. Commande bipolaire.

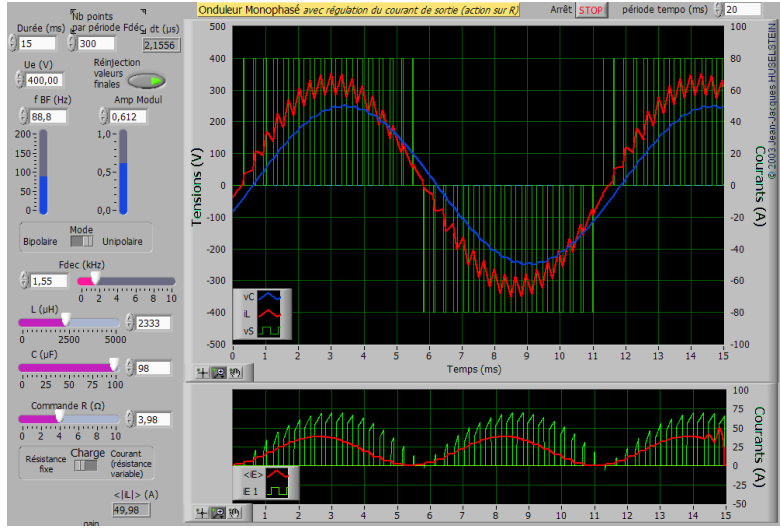

Fig. 10 : Onduleur monophasé. Commande unipolaire

\section{REDRESSEURS}

4.1 Redresseur monophasé sur charge capacitive L'étude du redressement sur charge capacitive est difficile du point de vue théorique si l'on souhaite ne pas déformer la réalité et bien tenir compte des différents éléments en jeu, notamment les imperfections du transformateur (résistance série et inductance de fuite) ainsi que les imperfections des diodes (chute de tension). Ce VI de simulation permet de bien mettre en évidence les effets de ces imperfections sur la forme, la valeur efficace et les harmoniques générées par ce type de redressement très répandu.

On s'intéressera bien sûr aussi à l'influence de la valeur de la capacité de filtrage. La charge peut être modélisée par une résistance ou bien par une source absorbant une puissance constante comme c'est souvent le cas lorsque le redresseur est suivi d'un convertisseur à découpage avec régulation de la tension de sortie.

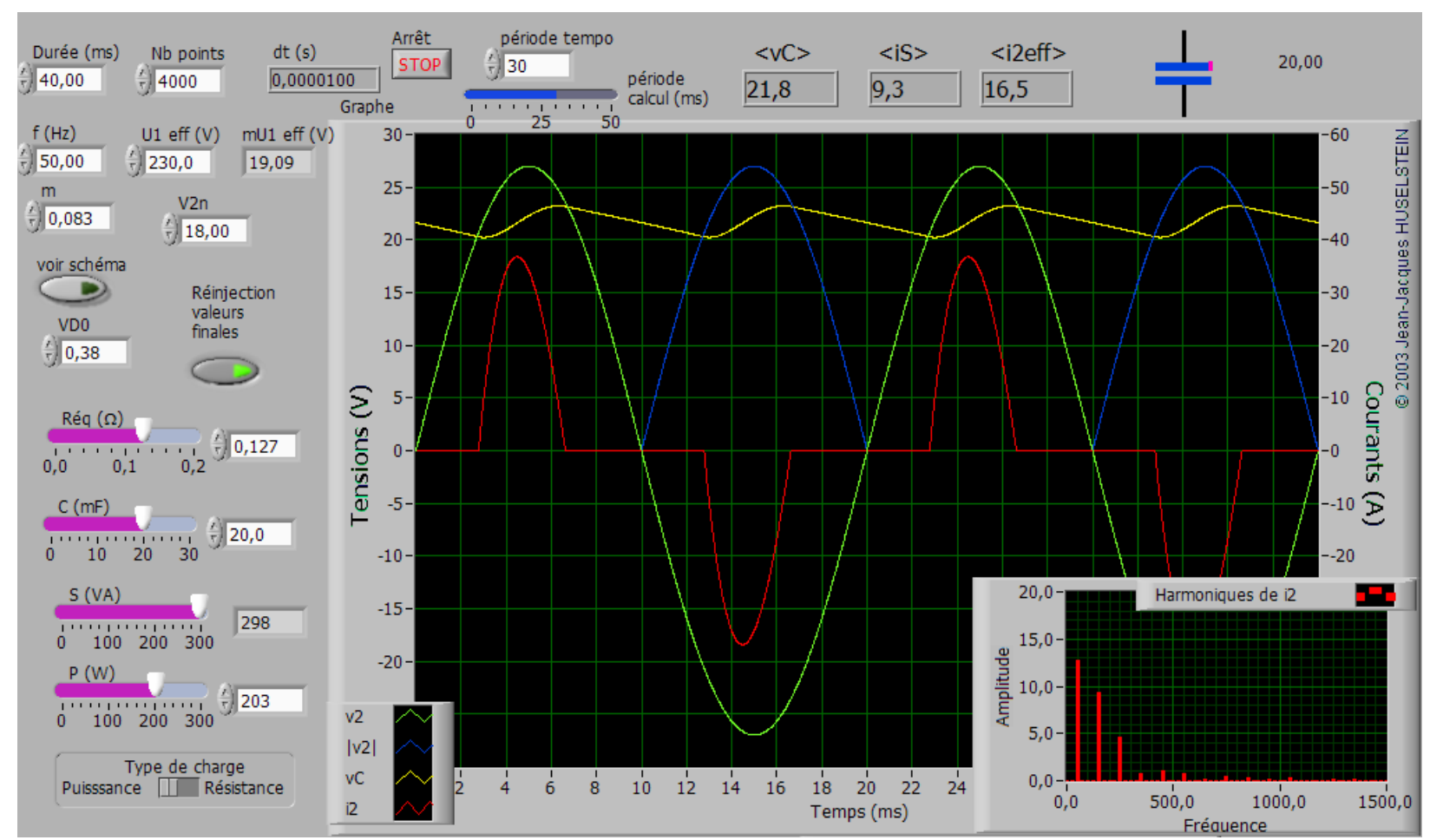

Fig. 11 : Redresseur monophasé sur charge capacitive. 


\section{UTILISATION PAR LES ETUDIANTS}

\subsection{Mise à disposition des étudiants}

La majeure partie des VI de simulation qui ont été réalisées sont mis à disposition des étudiants sur la page web :

http://www.univ-montp2.fr/ lie/Simul_EP_LabVIEW.html

\subsection{Coût logiciel et licences}

LabVIEW est un logiciel relativement onéreux, mais les applications présentées ici ne nécessitent pas l'installation de LabVIEW. Seul le moteur d'exécution LabVIEW, gratuit, est nécessaire. Il permet d'exécuter, mais sans les modifier, toutes les applications LabVIEW, donc les VI de simulation présentées ici. Un programme d'installation de ce moteur d'exécution et disponible avec les applications de simulation sur le site internet. National Instrument autorise la distribution légale et gratuite de ce moteur d'exécution.

\subsection{Licence étudiant LabVIEW}

Au delà de l'utilisation simple de ces applications de simulation, les étudiants intéressés par la programmation LabVIEW peuvent bénéficier d'un jeu de CD d'installation avec une licence gratuite. Il leur suffit pour cela d'en faire la demande sur le site de National Instrument.

\section{CONCLUSION}

Cet article présente des applications de simulations temporelles interactives de circuits de l'électronique de puissance destinées à une utilisation pédagogique. Nous avons, sur deux années consécutives, pu les utiliser avec satisfactions en cours pour illustrer de manière «vivante » les fonctionnement des certains convertisseurs de l'électronique de puissance en complément des «diaporamas» traditionnels. Les étudiants, et surtout certains d'entres eux plus particulièrement intéressés, ont pu profiter de la mise à disposition de ces applications pour continuer les simulations chez eux.

Malgré un procédé de calcul très simple les résultats de simulation sont très précis et très rapides (il en serait sûrement différemment si les circuits étaient plus complexes).

Il pourrait sembler regrettable à certains qu'il ne soit pas possible de modifier les circuits sans reprogrammer l'application mais ceci serait une utilisation totalement différente de l'objectif d'applications pédagogiques que nous nous étions fixé.

\section{Bibliographie}

1. Drofenik U., Kolar J.W.: Interactive Power Electronics Seminar (iPES) - A Web-Based Introductory Power Electronics Course Employing Java-Applets. Proceedings of the 33rd IEEE Power Electronics Specialists Conference, Cairns, Australia, June 23 - 27, Vol. 2, pp. 443 - 448 (2002).
2. Drofenik U., Kolar J.W. : Teaching Basics of Inductive Power Components Using Interactive Java Applets Performing FEM-Based On-Line Calculation of the Magnetic Flux Distribution. Proceedings of the 10th International Power Electronics and Motion Control Conference, Dubrovnik, Croatia, Sept. 9 - 11, CD-ROM, ISBN: 953-184-047-4 (2002).

3. IPES Circuits site web http://www.ipes.ethz.ch/

4. C. GLAIZE, J.-J. HUSELSTEIN, "Nouveaux outils pour l'enseignement des énergies renouvelables" CETSIS 2003, Toulouse, 13 \& 14 Novembre 2003, pp. 185-188.

5. National Instruments site web http://www.ni.com/.

6. http://www.univmontp2.fr/ lie/Simul_EP_LabVIEW.html 